\title{
In Vitro Biomechanical Strain Regulation of Fibroblast Wound Healing
}

Thanh V. Cao, BS

Michael R. Hicks, BS

Paul R. Standley, PhD

From the Department of Basic Medical Sciences at the University of Arizona College of Medicine-Phoenix (Mr Cao and Dr Standley) and the Department of Molecular and Cell Biology at Arizona State University in Tempe (Mr Hicks).

Financial Disclosures: None reported.

Support: Funding for these studies came from the National Institutes of Health's National Center for Complementary and Alternative Medicine PO-1AT2023 and the Arizona Biomedical Research Collaborative.

Address correspondence to Paul R. Standley, PhD, University of Arizona College of Medicine-Phoenix, HSEB, Room B558, 435 N 5th St, Phoenix, AZ 85004-2157.

E-mail: standley@email .arizona.edu Submitted

September 17, 2012; final revision received August 9, 2013; accepted

September 11, 2013.
Context: Strain-directed therapy such as vacuum compression and manual manipulative therapies are clinically effective, but their cellular and molecular mechanisms are not well understood.

Objective: To determine the effects of modeled myofascial release (MFR) on fibroblast wound healing and to investigate the potential role of nitric oxide (NO) in mediating these responses.

Methods: Using an in vitro scratch wound strain model, the authors investigated human fibroblast wound healing characteristics in response to injurious repetitive motion strain (RMS) and MFR. Secretion of NO was induced with interleukin- $1 \beta$ and sodium nitroprusside and inhibited with $\mathrm{NO}$ synthase inhibitor $\mathrm{L}-\mathrm{N}^{\mathrm{G}}$-monomethyl arginine citrate (L-NMMA) to determine the effects of NO on wound healing. Protein microarray was also performed to evaluate the expression of intracellular protein and activation of protein kinase $\mathrm{G}(\mathrm{PKG})$, extracellular signal-regulated kinase (ERK1/2), protein kinase $\mathrm{C}$ (PKC), and phosphoinositide 3-kinase (PI3K), the downstream effectors in the NO pathway.

Results: Fibroblasts that received RMS resulted in reduced wound closure rates (vs nonstrain, $P<.05$ ), which are partially attenuated by a single dose of MFR. Interleukin-1 $\beta$ and exogenous NO did not appear to have an effect on nonstrained fibroblast wound healing. However, strained fibroblasts appeared to express increased sensitivity to NO. The authors also observed a $12.2 \%$ increase in NO secretion, an increase in PKG activation, and a downregulation of PKC and PI3K inhibitory domain in the combined strain group.

Conclusion: If clinically translatable, these data suggest that mechanical strain such as vacuum compression therapy and manual manipulative therapy may modify PKC and PI3K to sensitize fibroblasts to $\mathrm{NO}$ and improve wound healing by promoting cell proliferation and migration by means of PKC and PKG signaling.

J Am Osteopath Assoc. 2013;113(11):806-818 doi:10.7556/jaoa.2013.056

0 train-applied therapies have been shown to be clinically efficacious in treating various soft tissue injuries. Treatment such as vacuum compression therapy has been widely reported to promote healing of chronic wounds associated with ischemic ulcers and diabetic foot ulcers. ${ }^{1-4}$ Other clinical studies of manual manipulative therapy have been shown to be effective in improving function, alleviating pain, and reducing risk of reoccurring injuries to the affected area. ${ }^{5}$ These treatment modalities are used to manage a variety of ailments, and their strain parameters share similar 
mechanical characteristics consisting of multiple myofascial release (MFR) patterns held for 60 to 90 seconds. Evidence from these and other clinical studies suggests that specific mechanical strain patterns may have direct implications in wound healing modification. ${ }^{6,7}$

During the proliferative and remodeling phases of wound healing, activation of fibroblasts stimulates secretions of necessary proinflammatory cytokines and extracellular matrix proteins, enhancing proliferation and migration, which when combined contribute to promoting tissue and cell repair. ${ }^{89}$ Activation of the inducible isoform of nitric oxide (NO) synthase in fibroblasts and consequent $\mathrm{NO}$ synthesis and secretion during the proliferative phase of wound healing is also well documented. ${ }^{10,11}$ Interestingly, biomechanical strain has been shown to be a potent activator of these multifunctional fibroblasts. ${ }^{12,13} \mathrm{We}$ have shown that strain upregulates interleukin (IL)-1 $\beta$, which is known to activate inducible NO synthase and subsequent NO secretions. Although the exact mechanism of NO contributing to wound healing remains elusive, it is known to regulate fibroblast proliferation, apoptosis, and migration ${ }^{14-16}$ by means of cyclic guanosine monophosphate (cGMP)-dependent kinase. ${ }^{17}$

We have previously modeled repetitive motion strain (RMS) (8 hours, 10\% $\mathrm{L}_{0}$ ) and manual manipulative techniques (MFR: 60 seconds $6 \% \mathrm{~L}_{0}$ ) in vitro to investigate the cellular and molecular effects of biomechanical strain on human fibroblasts. ${ }^{18}$ The MFR parameters for these in vitro strain models were empirically determined by videomorphometric analyses of clinically applied manual manipulative therapy, as we have reported elsewhere. ${ }^{18}$ Repetitive motion strain parameters were originally based on an 8-hour work day observing a roofer manually hammering nails. ${ }^{18,19}$ We found that RMS caused decreased fibroblast proliferation, increased apoptosis, upregulation of several inflammatory cytokines (ie, IL-1 $\alpha$, IL-1 $\beta$, IL-2, IL-3, IL-6, and IL-16), and induction of morphologic changes in actin architecture. ${ }^{18,20,21}$ Our MFR model was unable to reproduce this response by itself; when applied after RMS, however, the antiproliferative and inflammatory responses were suppressed. Our results showed that fibroblasts simultaneously serve roles as mechanical sensors and transmitters of force in manners that may potentially promote wound healing.

The objective of the present study was to assess in vitro fibroblast wound closure capabilities in response to various biomechanical strain patterns. To address this aim we used our previously reported in vitro strain models combined with a well-documented scratch wound assay ${ }^{22,23}$ to investigate potential effects of strain on fibroblast wound healing. We hypothesized that fibroblasts may transpose mechanical stimuli to adapt specifically to their biomechanical environment in manners that enhance wound healing. This effect may be partly a result of soluble mediators released by fibroblasts such as IL- $1 \beta$, which is known to activate inducible NO synthase and subsequent NO secretions to regulate wound healing through cGMPdependent kinase pathways (ie, protein kinase G [PKG], phosphoinositide 3-kinase [PI3K], and extracellular signal-regulated kinase [ERK1/2]). Results from these studies will build on cellular evidence describing favorable fibroblast wound healing after mechanical strain.

\section{Methods}

The present in vitro study was conducted from March 2010 to October 2011.

\section{Cell Culture}

Normal human dermal fibroblasts obtained from Cambrex Laboratories were cultured in Dulbecco's modified Eagles Medium supplemented with 2\% fetal bovine serum and $1 \%$ penicillin-streptomycin at $37^{\circ} \mathrm{C}, 5 \% \mathrm{CO}_{2}$, and $100 \%$ humidity. The medium was replaced every other day with fresh, prewarmed growth medium. Subconfluent cultures (acquired in 7 to 10 days) were passaged at a ratio of 1:3; all experiments used cell passages between 4 and 12 . 


\section{In Vitro Strain Apparatus}

All strain profiles were conducted using the Flexercell FX-4000 Tension Plus System (Flexcell International Corp). This apparatus is a computer-based system that uses vacuum pressure to strain cells adhered to flexible collagen-coated membranes arranged in a format of 6 wells per plate. The deformation of the collagen causes the attached fibroblasts to similarly deform. Programming the strain magnitude, duration, direction, and frequency of the negative pressure created the desired profiles. ${ }^{20}$

\section{Strain Profiles and Scratch Wound Assay}

Normal human dermal fibroblasts were seeded at a concentration of $1 \times 10^{5}$ cells/well onto collagen I-coated Bioflex plates. During protocol development, we observed that fibroblasts undergo growth arrest on this substrate once full confluence is reached. Therefore, once the cultures reached $70 \%$ to $80 \%$ confluence (approximately 24 hours after seeding), the growth medium was replaced with a reduced-serum medium ( $0.2 \%$ fetal bovine serum) to induce quiescence. After an additional 24 hours, a modeled scratch wound was applied using a sterile $1000 \mu \mathrm{L}$ pipet tip to remove all cells from the elastomeric substrate within this area. This process produced a "wound" area
$(2 \mathrm{~mm} \times 25 \mathrm{~mm})$ of approximately $50 \mathrm{~mm}^{2}$ completely devoid of cells. Cellular debris was removed along with the medium by rinsing the fibroblast cultures with sterile phosphate buffer saline (Sigma Aldrich). Fresh growth medium was then added to each well, and the wound cultures were then subjected to 1 of the 4 strain paradigms: no strain (control), RMS, MFR, and combined treatment (RMS+MFR) as shown in Figure $1 \mathrm{~A}$.

Strain magnitudes, frequencies, and durations of our RMS model were based on an 8-hour work day observing a roofer manually and repeatedly hammering nails. ${ }^{19}$ This RMS injury paradigm is a cyclic strain that stretches samples $10 \%$ beyond the initial resting length at $1.6 \mathrm{~Hz}$ for 8 hours. This paradigm is intended to model scenarios such as the working class or athletes who ignore proper wound care and continue to engage in repetitive labor or physical competitions (Figure 1B). Our MFR models consist of a slow-loading, 60-second acyclic strain that stretches samples to $6.6 \%$ beyond the initial resting length. These parameters were empirically determined by videomorphometric analyses of clinically applied manual manipulative therapy and reflect treatment immediately after injury (Figure $1 C$ ). We have reported on this modeled manual manipulative therapy
A

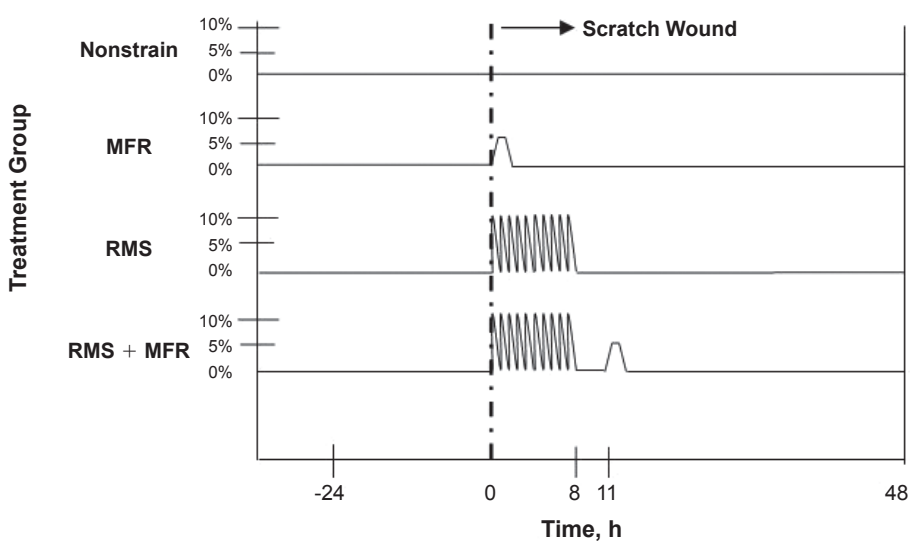

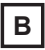

Slope: $33.3 \% / \mathrm{s}$
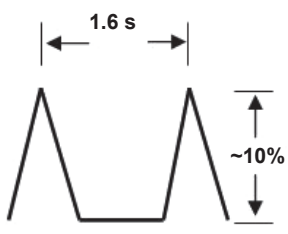

RMS
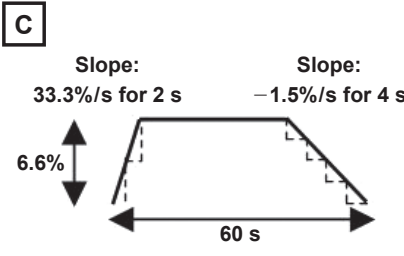

MFR

Figure 1.

(A) Strain profiles and experimental timelines for the 4 treatment groups used: nonstrain, myofascial release (MFR), repetitive motion strain (RMS), and RMS + MFR. Illustrations of (B) a single RMS cycle and (C) a complete 60 -second cycle of MFR. Images $B$ and $C$ reprinted from Meltzer et $\mathrm{al}^{18}$ with permission from Elsevier. 
previously. ${ }^{18,19}$ The combined strain group represents a scenario of a patient seeking MFR treatment 3 hours after a potential re-aggravated injury resulting from continued overuse of the affected area. We recognize that various limitations exist with using such in vitro models to correlate with wound healing and in vivo biomechanics. For example, we do not account for other cell types that also contribute to wound healing, such as neutrophils, lymphocytes, and macrophages, or the biomechanical effects of normal tissue tension and induced inflammation in response to injury. Evaluating such aspects was beyond the scope of our interests, and this in vitro wound model provides us with a reproducible and controllable environment to investigate fibroblast wound healing behavior in response to biomechanical strain.

\section{Photomicrography and}

\section{Wound Healing Rate Quantification}

For each wound construct, digital images were captured at magnification $\times 40$, which produced a $7-\mathrm{mm}^{2}$ maximum viewable wound area (Figure $2 A$ ). Repeated measures were acquired of the same location from each construct immediately and 8, 24, and 48 hours after wounding using premarked grids placed on the undersides of the Bioflex wells. Phase contrast images were captured using an IX71 Olympus inverted microscope and DP71 camera (Olympus America Inc). Wound margins were determined and outlined by a single observer (T.C.) using Adobe Photoshop CS2 version 9.0 (Adobe System Inc). Digital images were then analyzed using Image J 1.40g (National Institutes of Health) and Cell Profiler (Broad Institute) to measure spatial changes (in square millimeters) to the area devoid of cells (remaining wound area; Figure 2A, Figure 2B, and Figure 2C). Fibroblast wound closure rates were determined as the absolute value of the slope $(\mathrm{m})$ described by the linearly regressed line of the wound area (Figure 2D).

\section{Conditioned Media Cross Sampling}

Recipient nonstrained wound constructs were treated with conditioned media (CM) collected from wound constructs previously exposed to the 4 strain paradigms (donors: nonstrain, MFR, RMS, and RMS+MFR) described above. Conditioned media were collected 48
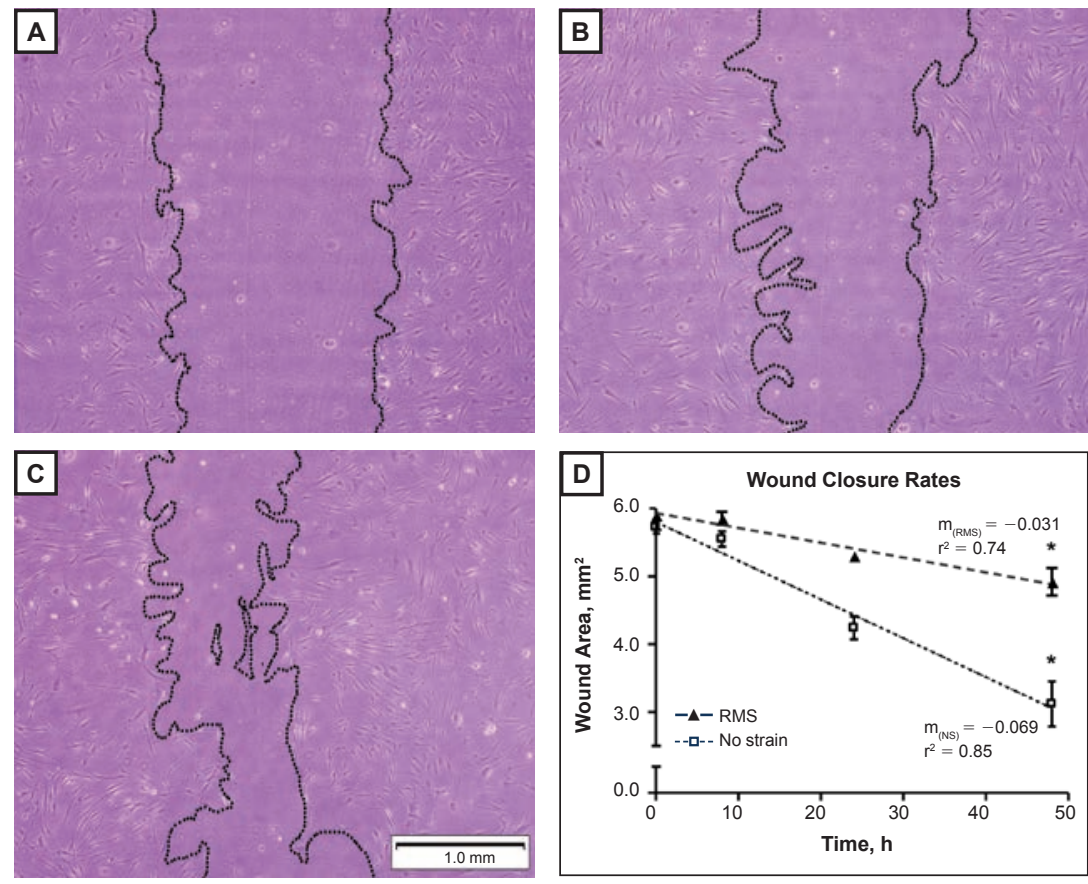

Figure 2.

Representative photomicrographs of nonstrain human fibroblast constructs $(A)$ immediately (time $=0$ ), (B) 24 hours, and (C) 48 hours after wounding. (D) Changes to the wound area over a 48-hour period. Wound healing rate is equal to the absolute value of the slope $(m)$ described by the linearly regressed line $(n=5)$. All images were captured at magnification $\times 40$, and the scale bar indicates $1.0 \mathrm{~mm}$. Abbreviations: NS, no strain; RMS, repetitive motion strain. 
hours after wounding and treatment with respective strain groups. Wound closure rates were then monitored within the recipient constructs by the method described previously to determine the potential roles of strain-regulated fibroblast-derived soluble mediators in wound healing rates.

\section{NO Measurements}

Conditioned media from each of the 4 strain groups were collected and stored at $-80^{\circ} \mathrm{C}$. The level of $\mathrm{NO}$ secretion was quantified using a nitrate/nitrite fluorometric assay (Cayman Chemical Company). Nitric oxide measurements are reported as a percentage change from the nonstrained control. Wound healing was also determined for strained and nonstrained wounded cultures treated with exogenous NO by incubation with $0,5,10$, or $25 \mu \mathrm{M}$ of the NO donor sodium nitroprusside (Sigma Alderich). In a subset of experiments, strained and nonstrained wounded fibroblast constructs were also treated with the NO synthase inhibitor $\mathrm{L}-\mathrm{N}^{\mathrm{G}}$-monomethyl arginine citrate (L-NMMA; $100 \mathrm{nM}$ ) and assessed for wound closure.

\section{Effects of Exogenous IL-1 $\beta$ and IL-1 $\beta$ Neutralizing Antibody on Fibroblast Wound Closure}

Potential roles of IL-1 $\beta$ in mediating fibroblast wound closure were evaluated by treating nonstrained wound constructs with $10 \mathrm{ng} / \mathrm{mL}$ IL-1 $\beta$ (R\&D Systems) for 48 hours. Effectiveness of IL- $1 \beta$ was determined by measuring IL-1 $\beta$ 's known ability to stimulate fibroblast NO secretion. In addition, fibroblast basal and strain-induced IL-1 $\beta$ secretion was neutralized using the recommended dose of $10 \mathrm{ng} / \mathrm{mL}$ of IL- $1 \beta$ mouse immunoglobulin G antibody (R\&D Systems).

\section{Intracellular Protein Microarray}

Antibody microarrays services from Kinexus Bioinformatics Corporation were used to track potential changes in 800 intracellular signaling proteins. The assay used individual antibodies specific for various protein kinases, cell cycle proteins, stress, apoptosis, and protein phosphatases. Each sample was pooled from 21 individual wells 48 hours after wounding and tested pairwise. Lysates were prepared according to the company's protocol and assayed by Kinexus Bioinformatics Corporation in a blinded fashion. For brevity, only signaling proteins involved in the NO pathway are shown as a percentage change from control ( $n=2$ per strain group).

\section{Statistical Analysis}

Wound healing data were collected and assessed for each strain paradigm in 4 to 5 experiments with triplicate testing ( $\mathrm{N}=12$ to15 per group). Conditioned media from each strain profile was collectively pooled for the CM cross test. Media crossover experiments were performed in 2 experiments, each in duplicate ( $\mathrm{N}=4$ per group). Nitric oxide measurements, NO synthase inhibitor measurements, and IL- $1 \beta$ induction experiments were conducted over 2 separate experiments, each with 3 to 4 replicate wells per treatment group ( $n=6$ to 7$)$. Data were calculated as mean (standard deviation), and the 4 treatment groups were compared by unpaired, 2-tailed $t$ tests using Prism 4.03 (GraphPad Software, Inc). Group means with $P<.05$ were considered to be statistically significantly different.

\section{Results}

\section{RMS Impairs Wound Closure Rate}

The change in wound area over the span of 48 hours exhibited a linear relationship (Figure 2D). Forty-eight hours after wounding, nonstrained wound constructs yielded the fastest wound closure rates $(0.066$ [0.018] $\mathrm{mm}^{2} / \mathrm{h}, \mathrm{n}=15$ ), as described by the linearly regressed line. Cells treated with 60 seconds MFR showed no significant difference in wound closure rates when compared with control cells $\left(0.064[0.024] \mathrm{mm}^{2} / \mathrm{h}, \mathrm{n}=12\right.$, $P>.05)$. However, groups strained with 8 hours of RMS exhibited a $67 \%\left(0.022[0.016] \mathrm{mm}^{2} / \mathrm{h}, \mathrm{n}=14, P<.0001\right)$ decrease in wound closure rate as compared with the nonstrained control group. Fibroblasts treated with combined RMS +MFR resulted in a 39\% decrease $(0.040$ $\left.[0.012] \mathrm{mm}^{2} / \mathrm{h}, \mathrm{n}=12, P<.05\right)$ in wound closure rate as compared with control fibroblasts. Compared with the 
RMS group, MFR improved RMS-induced attenuation of wound closure rate by $181 \%$. These data are summarized in Figure $3 \mathrm{~A}$.

\section{From Cyclically Strained Fibroblasts Modifies Wound Closure Rates in Nonstrained Fibroblast Constructs}

Conditioned media crossover experiments were performed to determine whether biomechanical strain induced fibroblasts to secrete soluble mediators that can affect wound healing in the absence of strain. In these experiments, donor wound constructs were subjected to either no strain or 1 of 3 strain paradigms (MFR, RMS, or RMS+MFR). Wound closure rates were noted and recorded from these groups (Figure $3 A$ ). Additionally, the $\mathrm{CM}$ from all groups were saved and used in the crossover design.

Recipient wound constructs were not biomechanically strained. Instead, they were treated with the previously saved CM taken from the donor constructs described above. After 48 hours, wound closure rates were noted and recorded from these groups (Figure 3B).

There were no statistically significant differences in wound closure rates among groups cultured with $\mathrm{CM}$ from nonstrained fibroblast and those that received fresh growth media [data not shown] (fresh media: 0.061 $\mathrm{mm}^{2} / \mathrm{h}, \mathrm{n}=9$; nonstrained CM: $0.053[0.011] \mathrm{mm}^{2} / \mathrm{h}$, $\mathrm{n}=4 ; P>.05)$. Comparisons of wound closure rates of MFR CM and RMS+MFR CM to nonstrained CM were also not statistically significant. However, CM from RMS-treated fibroblasts was sufficient to impair wound closure by $30.2 \%$ in nonstrained fibroblast constructs (RMS CM: 0.037 [0.006] $\mathrm{mm}^{2} / \mathrm{h}, \mathrm{n}=4, P<.05$ ) when compared with nonstrained CM (Figure 3B).

\section{NO Induction by IL-1 $\beta$ and Its Effects on Wound Closure}

Interleukin- $1 \beta$ is 1 of the many cytokines that is upregulated by biomechanical strain with known roles in regulating wound healing and $\mathrm{NO}$ secretions. To investigate IL- $1 \beta$ as a potential mechanism for wound healing inhibition in our CM crossover study, we treated the non-
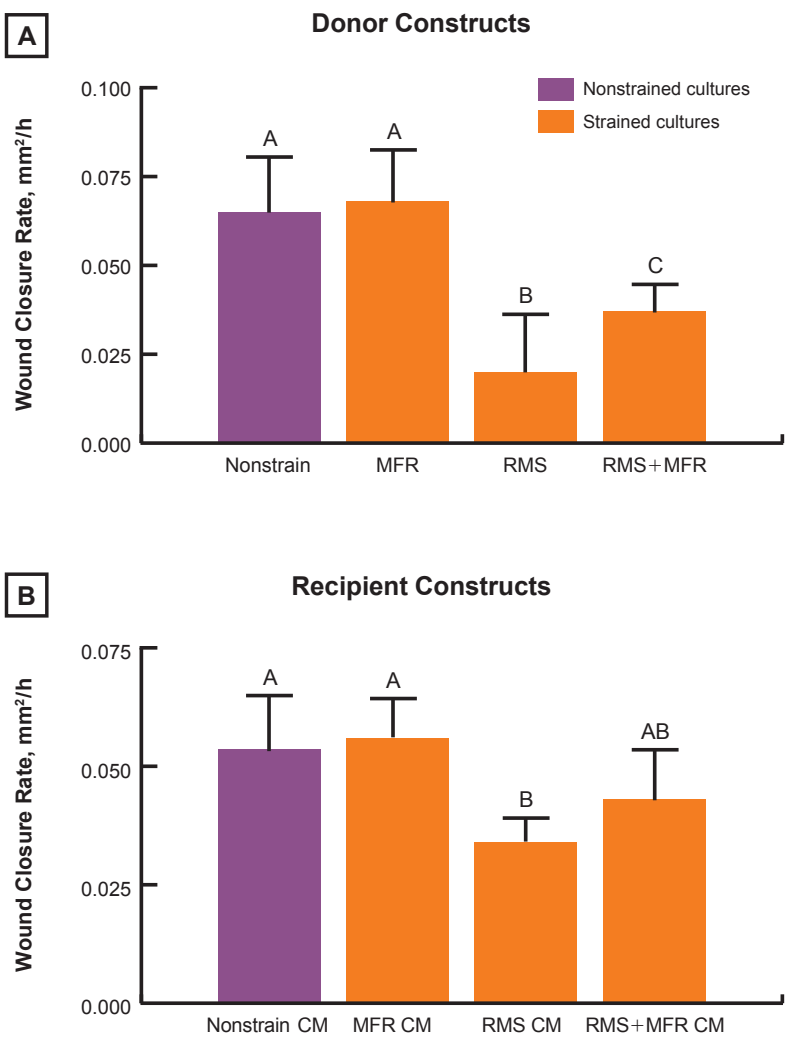

Figure 3.

(A) Fibroblast wound closure rates in donor constructs in response to modeled strain paradigms ( $n=12$ to 15$)$. (B) Nonstrained fibroblast recipient constructs were treated with conditioned media (CM) from previously strained (repetitive motion strain [RMS], myofascial release [MFR], and RMS+MFR) and nonstrained fibroblast cultures and assessed for wound closure rates $(n=4)$. Bars labeled with different letters indicate a significant difference between the groups $(P<.05)$ : bar $\mathrm{B}$ indicates significant decrease in wound healing rates compared with bar $A$ (nonstrain and MFR) and bar C (RMS +MFR). Bar C indicates a significant decrease in wound healing rates compared with bar $A$ (nonstrain and MFR).

strained wounded fibroblast construct with exogenous IL-1 $\beta$. The addition of human recombinant IL-1 $\beta$ (10 ng/ $\mathrm{mL})$ significantly induced NO secretion when measured 48 hours after wounding $(P<.05)$. Nonstrained fibroblast wound constructs treated with $100 \mathrm{nM}$ of L-NMMA did not display altered NO secretion compared with control 
fibroblasts. However, when L-NMMA was added concomitantly with IL-1 $\beta$, NO secretion was significantly suppressed compared with IL-1 $\beta$ treatment alone (Figure $4 A$ ). None of these treatments had any statistically significant impact on nonstrained fibroblast wound closure rates (Figure 4B).

\section{Biomechanical Strain Induces No Secretion}

Activation of inducible NO synthase and subsequent increase in NO secretion can be directly enhanced by biomechanical strain. Wound constructs treated with RMS + MFR exhibited the highest NO secretion, which increased by $12.2 \%(P<.05, \mathrm{n}=7)$ when compared with nonstrained wound constructs (Figure $5 A$ ). Nitric oxide secretion was not significantly modified in the MFR or RMS treatment constructs. Inhibiting NO synthase activity with $100 \mathrm{nM} \mathrm{L-NMMA}$ did not affect wound healing rates in the nonstrain control, RMS, or MFR groups. However, in our RMS +MFR group, in which we observed the highest NO secretions, equivalent NO inhibition resulted in $22 \%$ decreased wound healing rates compared with the untreated RMS+MFR group (Figure $5 B ; \mathrm{n}=6, P<.05$ ).

\section{NO Affects Fibroblast Wound Closure}

\section{in a Strain-Dependent Manner}

We used sodium nitroprusside (SNP), an NO-donating compound, to test the direct effect of $\mathrm{NO}$ on strained and nonstrained fibroblast wound healing. For every $1 \mu \mathrm{M}$ of SNP added to nonstrained control wound constructs, we observed a $0.63 \mu \mathrm{M}$ increase in measurable NO (Figure $6 A) 48$ hours after treatment. Quantification of wound healing in nonstrained wound constructs using these concentrations of SNP revealed no statistically significant differences among any treatment groups (Figure $6 B)$. In our RMS group, we found that $5 \mu \mathrm{M}$ SNP accelerated wound closure compared with no treatment, whereas 10 and $25 \mu \mathrm{M}$ revealed no change in wound closure rates compared with no treatment (Figure 6C). In our RMS+MFR group, we observed statistically significant decreases in wound closure rates at all concentrations of SNP tested (Figure 6D).

\section{Effects of Strain on NO Pathway Intermediates}

Biomechanical strain ability to alter fibroblast sensitivity to NO levels suggested that there may be potential regulation in the expression and phosphorylation of downstream intracellular proteins involved in the $\mathrm{NO}$ pathway. Protein microarray results indicated that $\mathrm{PKC} \alpha / \beta 2^{\mathrm{T} 638 / \mathrm{T} 641}$ phosphorylation was upregulated by $633 \%$ in our RMS+MFR group compared with the RMS group. In addition, we found PKG1 $\alpha / \beta$ to be universally upregulated in all strain groups, whereas PKG1 serves to activate multiple branches in the NO-mediated proliferative and migratory pathways: PKG1 $\rightarrow$ RAF1 (prot-oncogene $\mathrm{c}-\mathrm{RAF}) \rightarrow \mathrm{ERK} 1 / 2$ and $\mathrm{PKG} 1 \rightarrow \mathrm{PI} 3 \mathrm{~K} \rightarrow \mathrm{Akt}$ (protein kinase B). We observed increased phosphorylation of

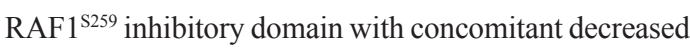
ERK1/2 ${ }^{\mathrm{T} 202+\mathrm{Y} 204 ; \mathrm{T} 185 / \mathrm{Y} 187}$ phosphorylation in all of our strain groups. Expression of PI3K was not substantially changed relative to the control group in any treatment group. However, in our RMS + MFR group, we observed a $44 \%$ upregulation in phosphorylation in PI3K regulatory subunit (PI3K p85/p55 ${ }^{\mathrm{Y} 476 / \mathrm{Y} 199}$ ). Detailed data are summarized in the Table.

\section{Comment}

In the present study, we investigated the differential effects of various biomechanical strain profiles on fibroblast wound healing by using an in vitro injury strain model. To our knowledge, the present study is the first to show that fibroblast wound closure responds uniquely to different biomechanical strain patterns. Strained fibroblasts exhibited increased sensitivity to changes in NO levels in which low concentrations of NO served to enhance wound healing rates. In addition, a single dose of MFR was sufficient to reverse the wound healing impairment induced by RMS, which may be potentially mediated by increased NO secretion and activation of the PKC and PKG pathways.

We found that RMS attenuated wound closure rates by $67 \%(P<.05)$. Myofascial release alone failed to modify closure rates when compared with control; how- 
ever, when applied after RMS, it enhanced wound healing (Figure 3A). An in vivo study conducted by Carpenter et $\mathrm{al}^{24}$ showed that rotator cuff injury, when coupled with overuse strain, prolonged healing times when compared with overuse alone. We observed similar results with our in vitro injury model indicating impaired wound healing in injury combined with RMS groups. Similarly, CM from RMS-treated fibroblasts was also sufficient to similarly affect wound closure rates in wounded, nonstrained fibroblast cultures. This response was not observed from any other CM crossover treatment groups (Figure 3B). These findings may be partially explained by other in vitro studies, which found that strain patterns of varying magnitude, duration, and direction promoted different responses in terms of fibroblast morphological changes, proliferation, apoptosis, signaling pathway, and secretions of inflammatory cytokines, ${ }^{18-21,25}$ all of which may in turn affect overall fibroblast wound healing rates. These data support the fact that RMS-treated fibroblasts secrete soluble mediators, whose efficacies are preserved despite a single freeze/ thaw cycle, which is capable of also inducing autocrine wound closure inhibition in the absence of strain in recipient constructs.

We hypothesized that NO may play a significant role in mechanotransduction for signaling wound healing. Although the exact roles of NO in wound healing remain elusive, it has been shown to regulate fibroblast proliferation, NO synthase induction (and subsequent NO secretion), differentiation, and apoptosis. ${ }^{26-28}$ Fibroblasts exhibit pleiotropic responses to NO in a concentration-dependent manner. For example, high concentrations of NO may serve as proapoptotic signaling molecules, ${ }^{15,29}$ whereas low NO concentrations stimulate fibroblast proliferation. ${ }^{16}$ Biomechanical strain and paracrine mediation by means of IL- $1 \beta$ are of the many factors that influence the formation of NO. ${ }^{13,30}$ From our previous study,,${ }^{19}$ we found IL-1 $\beta$ to be upregulated 4-fold in response to RMS. Interestingly, this same study also showed that MFR applied both alone and after RMS was effective in suppressing secretion of IL-1 $\beta$ and various other proinflammatory cytokines. ${ }^{19}$ This finding offers a possible explanation for the lack of wound
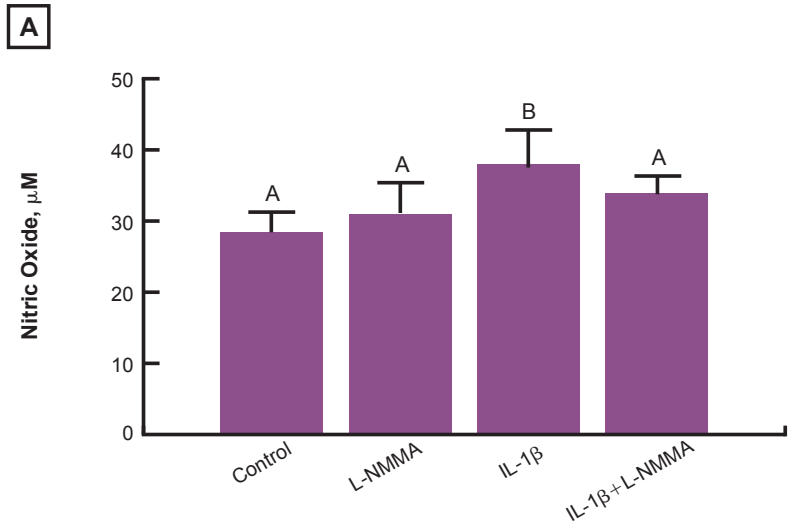

B

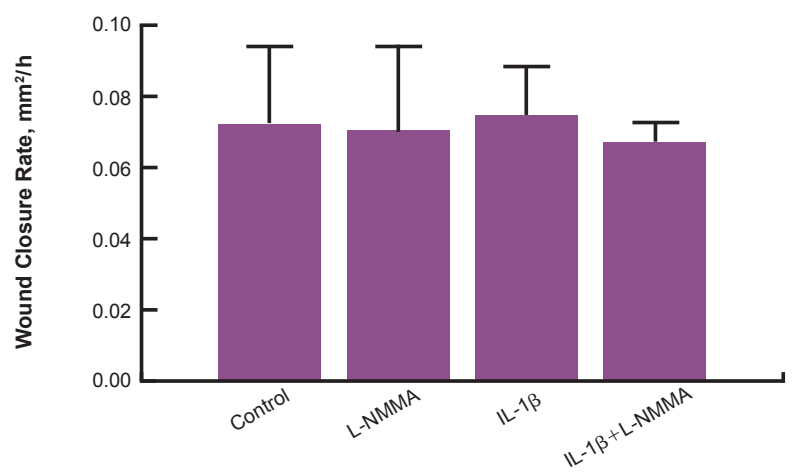

Figure 4.

Nitric oxide synthase induction and inhibition generated with recombinant human interleukin (IL)-1 $\beta(10 \mathrm{ng} / \mathrm{mL})$ and L-N ${ }^{\mathrm{G}}$-monomethyl arginine citrate (L-NMMA) (100 nM), respectively. (A) Nitric oxide secretion and $(B)$ wound closure rates 48 hours after wounding. Bars labeled with different letters indicate a significant difference between the groups ( $\mathrm{n}=5$ to 7$)(P<.05)$ : bar $\mathrm{B}$ indicates a significant increase in nitric oxide secretion compared with control, L-NMMA, and IL-1 $\beta+$ L-NMMA (bar A).

healing impairment from our MFR and RMS+MFR CM crossover group.

To investigate the effects of IL-1 $\beta$ on fibroblast wound healing, we treated nonstrained fibroblasts with $10 \mathrm{ng} / \mathrm{mL}$ of IL-1 $\beta$ which predictably resulted in increased NO secretion. ${ }^{30,31}$ Additionally, NO induction in response to IL- $1 \beta$ was effectively suppressed with the 


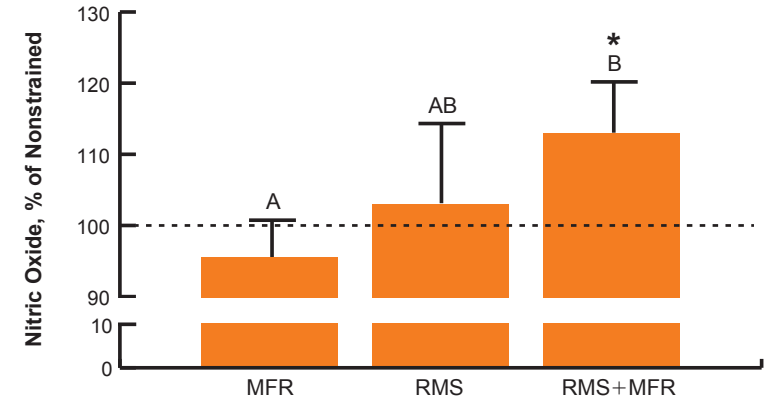

B

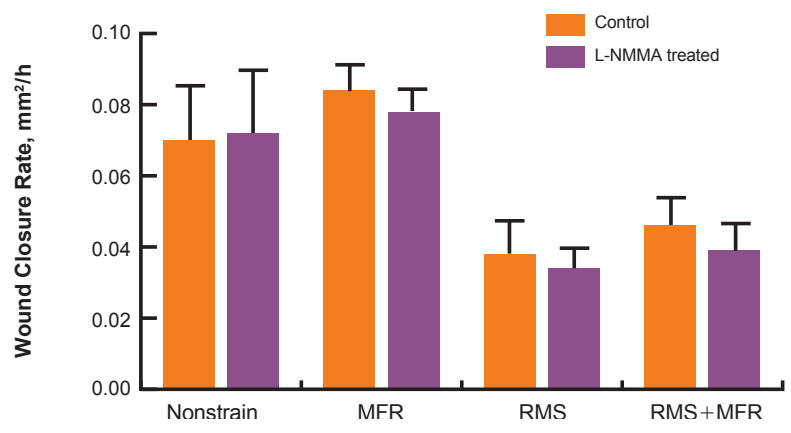

Figure 5 .

(A) Nitric oxide secretion measured as a percentage of nonstrained control in response to myofascial release (MFR), repetitive motion strain (RMS), and combined RMS + MFR treatments. (B) Wound closure rates in nonstrained and strained groups treated with and without L- $\mathrm{N}^{\mathrm{G}}$-monomethyl arginine citrate (L-NMMA) serve as treatment and control, respectively. Bars labeled with different letters indicate a significant difference between the groups $(P<.05, \mathrm{n}=6$ to 7$)$ : bar $B(R M S+M F R)$ indicates a significant increase of nitric oxide secretion compared with MFR (bar A). ${ }^{a} P<.05$ vs nonstrain control.

addition of $100 \mathrm{nM}$ L-NMMA (Figure 4A), proving the efficacy of this NO synthase inhibitor in our in vitro model. Assessment of wound closure in the presence of exogenous IL-1 $\beta$ revealed no differences among control and treatment groups (Figure 4B). We also treated strained and nonstrained fibroblast constructs with mouse IL-1 $\beta$ and found that neutralizing IL-1 $\beta$ did not have any effect on fibroblast wound closure (data not shown). These results indicate that IL- $1 \beta$ and its consequent increased NO secretions may not be sufficient to modify fibroblast wound closure (in nonstrained constructs). This finding, however, does not rule out other possibilities, including that IL-1 $\beta$ may simultaneously activate dual pathways to regulate NO-induced proliferation and IL-1 $\beta$-mediated apoptosis, ${ }^{31}$ thereby effectively balancing changes in observed wound closure rates.

Fibroblasts secrete basal levels of $\mathrm{NO}$, and neither MFR nor RMS alone significantly altered fibroblast NO secretion when compared with nonstrain control. In our combined treatment (RMS +MFR), we observed a $12.2 \%(P<.05)$ increase in NO secretion compared with baseline (Figure $5 A$ ). We have previously reported that RMS induces a fibroblast inflammatory response in vitro, and from these data we speculate that the ability for MFR to regulate NO secretions may require a proinflammatory state. Inhibition of NO synthese (using the previously proven effective dose of L-NMMA as described in Figure 4) in the RMS +MFR group led to a $22 \%$ reduction $(P<.05)$ in wound healing rate when compared with that in the untreated RMS +MFR group (Figure 5B, $P<.05)$. Equivalent NO inhibition in our nonstrain control, MFR, and RMS only groups did not have such an effect, therefore suggesting that MFR administered after RMS induced wound healing in an NO-dependent manner. Exogenous NO results indicate that wound closure rates are nonresponsive to changes in NO concentration from 0 to $25 \mu \mathrm{M}$ in our control group (Figure $6 B$ ). However, RMS-treated constructs resulted in increased wound healing rates with low level NO $(5 \mu \mathrm{M})$ and showed no change at higher NO concentrations (10-25 $\mu \mathrm{M}$; Figure $6 C)$. Fibroblasts that received combined strain treatment showed significant impairment at all increasing dosing of SNP (Figure 6D). The lack of response observed in our nonstrain in vitro fibroblast cultures suggest that (1) mechanically strained fibroblasts express increased sensitivity to changes in NO levels or (2) wound healing rates are optimal at specific low NO concentrations. The ability of MFR to induce NO secretion and enhance wound healing was only apparent when a condition of 
A

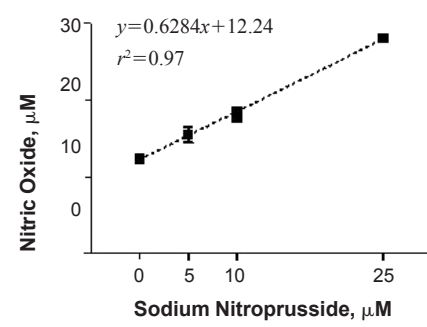

c

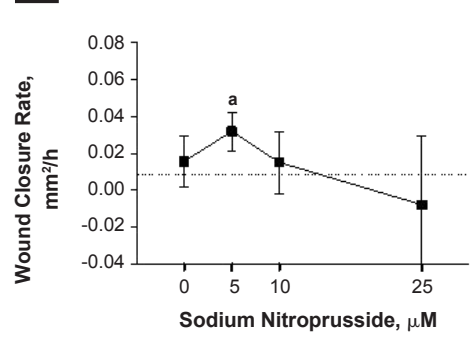

Control

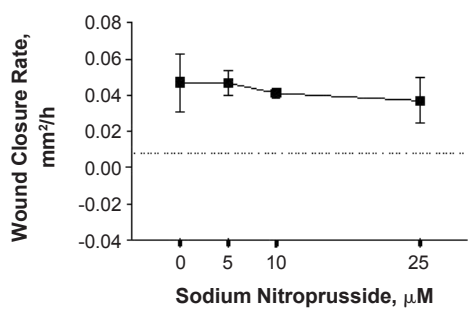

RMS+MFR

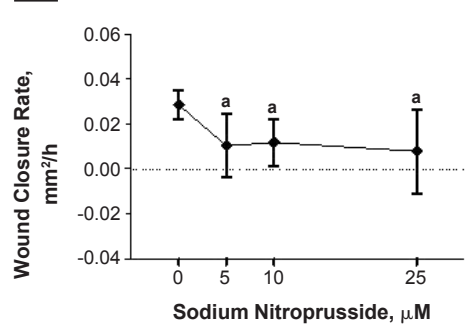

Figure 6.

(A) Quantifiable nitric oxide resulting from sodium nitroprusside additions (5-25 $\mu \mathrm{M})$. (B) Nonstrained control, (C) repetitive motion strain (RMS), and (D) RMS + myofascial release (MFR) fibroblast wound healing rates in response to various concentrations of the nitric oxide donor sodium nitroprusside $(n=6)$. Wound healing assessments were made 48 hours after wounding. ${ }^{a} P<.05$ vs nonstrain control.

impaired wound healing existed (injury+RMS). These data support that increased NO secretions may be 1 of the mechanisms by which a single dose of MFR improves wound healing after RMS.

Nitric oxide activates multiple wound closure-relevant pathways that directly regulate cellular processes such as cell proliferation, migration, differentiation, and apoptosis. Such intracellular proteins regulated by NO include protein kinase $\mathrm{C}$ and cGMP-dependent PKG. ${ }^{32,33}$ Protein microarray results indicate that although PKC expression was not different among any of the treatment groups (data not shown), we did observe a $633 \%$ increase in phosphorylation of $\mathrm{PKC} \alpha / \beta 2$ at threonine 638 in the RMS + MFR treatment compared with RMS alone. Treatments of RMS only and MFR only did not have such an effect. Although phosphorylation of this site is not essential for PKC activity, it serves to prolong activated PKC functionality by inhibiting dephosphorylation of the PKC catalytic domain. ${ }^{34}$ Treatments of RMS only also increased cGMP-dependent protein kinase of both PKG1 $\alpha$ and PKG1 $\beta$ subunits 48 hours after wounding. Application of MFR alone and after RMS served to further increase the expression of PKG1 $\alpha / \beta$ by approximately 5 - and 2-fold, respectively, when compared with RMS only. Nitric oxide-dependent PKG is known to promote the mitogenic response by activating the RAF1/ ERK1/2 pathway. ${ }^{35}$ However, activation of this pathway appears unlikely, as results indicate increased phosphorylation of the inhibitory domain at RAF $1^{\mathrm{S} 259}$, which prevents RAF1 membrane accumulation and activation. ${ }^{36}$ 
This result is consistent with our data, which indicate decreased ERK1/2 phosphorylation at the activation site $\mathrm{T} 202+\mathrm{Y} 204 / \mathrm{T} 185+\mathrm{Y} 187^{37,38}$ (Table). In addition, PKG has been shown to promote cell survival in response to stress by activating a PI3K/Akt-dependent response. ${ }^{39,40}$ Although we did not specifically look at Akt activity, we noted increased phosphorylation of the regulatory sub-

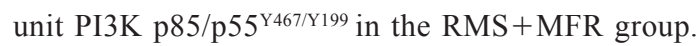
Phosphorylation of the regulatory subunit promotes assembly and activation of the PI3K signaling complex by removing the inhibitory effects of $\mathrm{p} 85 .{ }^{41}$ These data are preliminary but are consistent with our observed findings indicating altered fibroblast sensitivity to NO. Whether strain, NO, or both affect the regulatory subunits of PKC or PI3K remains unknown, but these results offer potential mechanistic approaches to describe the biomechanical roles in strain-induced wound healing.

\section{Conclusion}

The exact mechanism as to why RMS leads to impaired wound healing remains elusive. These data suggest that strained fibroblasts display increased sensitivity to changes in NO. Improved wound healing rates observed from a single MFR treatment after RMS may be the result of increased NO secretion, sensitivity, or both, leading to activation of the PI3K $\rightarrow$ Akt and PKC pathways. If clinically translatable, this finding suggests that improperly healed injuries that are reaggravated by continued overuse may hinder wound healing response. However, this impaired wound healing may benefit from treatments that use slow-loading MFR patterns such as those used by vacuum compression therapy and manual manipulative therapy. This wound closure model may be useful for further studies to identify cellular mechanisms linking biomechanical strain to wound repair and

Table.

Cellular Function and Percentage Change From Control in the Expression and Phosphorylation of Intracellular Proteins Involved in the Nitric Oxide Pathway

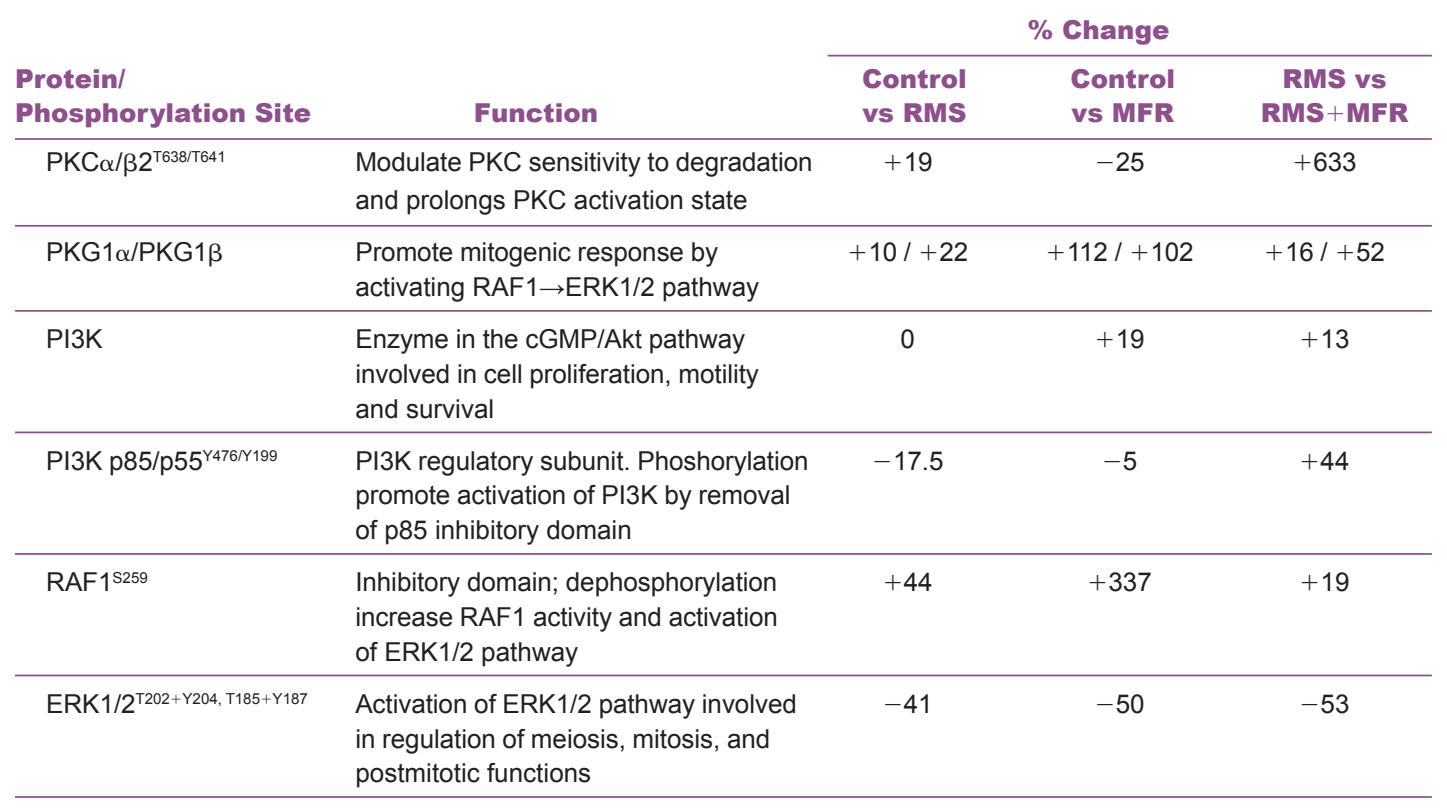

Abbreviations: ERK1/2, extracellular signal-regulated kinase; PI3K, phosphoinositide 3-kinase; PKC, protein kinase C; PKG, protein kinase G; RAF1, prot-oncogene c-RAF. 
providing evidence supporting manual therapeutic treatments in hastening wound repair (eg, vacuum compression therapy, manual manipulative therapy).

\section{Acknowledgments}

\author{
We thank David Campbell, BS, for his technical \\ assistance.
}

\section{References}

1. Akbari A, Moodi H, Ghiasi F, Sagheb HM, Rashidi H. Effects of vacuum-compression therapy on healing of diabetic foot ulcers: randomized controlled trial. J Rehabil Res Dev. 2007;44(5): 631-636. doi:10.1682/JRRD.2007.01.0002.

2. McCulloch JM Jr, Kemper CC. Vacuum-compression therapy for the treatment of an ischemic ulcer [case report]. Phys Ther. 1993;73(3):165-169. http://ptjournal.apta.org/content/73/3/165 .long. Accessed October 2, 2013.

3. Blume PA, Walters J, Payne W, Ayala J, Lantis J. Comparison of negative pressure wound therapy using vacuum-assisted closure with advanced moist wound therapy in the treatment of diabetic foot ulcers: a multicenter randomized controlled trial. Diabetes Care. 2008;31(4):631-636. http://care.diabetesjournals.org/content /31/4/631.full.pdf. Accessed October 2, 2013.

4. McNulty AK, Schmidt M, Feeley T, Kieswetter K. Effects of negative pressure wound therapy on fibroblast viability, chemotactic signaling, and proliferation in a provisional wound (fibrin) matrix. Wound Repair Regen. 2007;15(6):838-846.

5. Eisenhart AW, Gaeta TJ, Yens DP. Osteopathic manipulative treatment in the emergency department for patients with acute ankle injuries. J Am Osteopath Assoc. 2003;103(9):417-421. http://www.jaoa.org/content/103/9/417.full.pdf. Accessed October 2, 2013

6. Pietramaggiori G, Liu P, Scherer SS, et al. Tensile forces stimulate vascular remodeling and epidermal cell proliferation in living skin. Ann Surg. 2007;246(5):896-902.

7. Scherer SS, Pietramaggiori G, Mathews JC, Prsa MJ, Huang S, Orgill DP. The mechanism of action of the vacuum-assisted closure device. Plast Reconstr Surg. 2008;122(3):786-797.

8. Hübner G, Brauchle M, Smola H, Madlener M, Fässler R, Werner $\mathrm{S}$. Differential regulation of pro-inflammatory cytokines during wound healing in normal and glucocorticoid-treated mice. Cytokine. 1996;8(7):548-556.

9. Tettamanti G, Grimaldi A, Rinaldi L, et al. The multifunctional role of fibroblasts during wound healing in Hirudo medicinalis (Annelida, Hirudinea). Biol Cell. 2004;96(6):443-455. doi:10.1016/j.biolcel .2004.04.008.

10. Lee RH, Efron D, Tantry U, Barbul A. Nitric oxide in the healing wound: a time-course study. J Surg Res. 2001;101(1):104-108. doi:10.1006/jsre.2001.6261.
11. Stallmeyer B, Kämpfer H, Kolb N, Pfeilschifter J, Frank S The function of nitric oxide in wound repair: inhibition of inducible nitric oxide-synthase severely impairs wound reepithelialization. J Invest Dermatol. 1999;113(6):1090-1098. http://www.nature.com/jid/journal/v113/n6/pdf/5600567a.pdf. Accessed October 2, 2013.

12. Webb K, Hitchcock RW, Smeal RM, Li W, Gray SD, Tresco PA. Cyclic strain increases fibroblast proliferation, matrix accumulation, and elastic modulus of fibroblast-seeded polyurethane constructs. J Biomech. 2006;39(6):1136-1144. doi:10.1016/j.jbiomech .2004.08.026.

13. van Griensven M, Zeichen J, Skutek M, Barkhausen T, Krettek C, Bosch U. Cyclic mechanical strain induces NO production in human patellar tendon fibroblasts-a possible role for remodelling and pathological transformation. Exp Toxicol Pathol. 2003;54(4): 335-338. doi:10.1078/0940-2993-00268

14. Witte MB, Thornton FJ, Efron DT, Barbul A. Enhancement of fibroblast collagen synthesis by nitric oxide. Nitric Oxide. 2000;4(6):572-582. doi:10.1006/niox.2000.0307.

15. Raghuram N, Fortenberry JD, Owens ML, Brown LA. Effects of exogenous nitric oxide and hyperoxia on lung fibroblast viability and DNA fragmentation. Biochem Biophys Res Commun. 1999;262(3):685-691. doi:10.1006/bbrc.1999.1216.

16. Schaffer MR, Efron PA, Thornton FJ, Klingel K, Gross SS, Barbul A. Nitric oxide, an autocrine regulator of wound fibroblast synthetic function. J Immunol. 1997;158(5):2375-2381.

17. Dhaunsi GS, Ozand PT. Nitric oxide promotes mitogen-induced dna synthesis in human dermal fibroblasts through cGMP. Clin Exp Pharmacol Physiol. 2004;31(1-2):46-49. doi:10.1111 j.1440-1681.2004.03948.x.

18. Meltzer KR, Cao TV, Schad JF, King H, Stoll ST, Standley PR. In vitro modeling of repetitive motion injury and myofascial release. J Bodyw Mov Ther. 2010;14(2):162-171. doi:10.1016/ j.jbmt.2010.01.002.

19. Meltzer KR, Standley PR. Modeled repetitive motion strain and indirect osteopathic manipulative techniques in regulation of human fibroblast proliferation and interleukin secretion. J Am Osteopath Assoc. 2007;107(12):527-536. http://www.jaoa .org/content/107/12/527.full.pdf. Accessed October 2, 2013.

20. Eagan TS, Meltzer KR, Standley PR. Importance of strain direction in regulating human fibroblast proliferation and cytokine secretion: a useful in vitro model for soft tissue injury and manual medicine treatments. J Manipulative Physiol Ther. 2007;30(8):584-592. doi:10.1016/j.jmpt.2007.07.013.

21. Dodd JG, Good MM, Nguyen TL, Grigg AI, Batia LM, Standley PR. In vitro biophysical strain model for understanding mechanisms of osteopathic manipulative treatment. J Am Osteopath Assoc. 2006;106(3):157-166. http://www.jaoa.org/content/106/3/157.long. Accessed October 2, 2013

22. Yarrow JC, Perlman ZE, Westwood NJ, Mitchison TJ. A high-throughput cell migration assay using scratch wound healing, a comparison of image-based readout methods. BMC Biotechnol. 2004;4:21. doi:10.1186/1472-6750-4-21.

23. Liang CC, Park AY, Guan JL. In vitro scratch assay: a convenient and inexpensive method for analysis of cell migration in vitro. Nat Protoc. 2007:2(2):329-333, doi:10.1038/nprot.2007.30. 
24. Carpenter JE, Flanagan CL, Thomopoulos S, Yian EH, Soslowsky LJ. The effects of overuse combined with intrinsic or extrinsic alterations in an animal model of rotator cuff tendinosis. Am J Sports Med. 1998;26(6):801-807.

25. Nishimura K, Blume P, Ohgi S, Sumpio BE. Effect of different frequencies of tensile strain on human dermal fibroblast proliferation and survival. Wound Repair Regen. 2007;15(5): 646-656. doi:10.1111/j.1524-475X.2007.00295.x.

26. Vernet D, Ferrini MG, Valente EG, et al. Effect of nitric oxide on the differentiation of fibroblasts into myofibroblasts in the Peyronie's fibrotic plaque and in its rat model. Nitric Oxide. 2002;7(4):262-276. doi:10.1016/S1089-8603(02)00124-6.

27. Palmer JN, Hartogensis WE, Patten M, Fortuin FD, Long CS. Interleukin-1 $\beta$ induces cardiac myocyte growth but inhibits cardiac fibroblast proliferation in culture. J Clin Invest. 1995;95(6):2555-2564. doi:10.1172/JCl117956.

28. Jeong JG, Kim JM, Cho H, Hahn W, Yu SS, Kim S. Effects of IL-1ß on gene expression in human rheumatoid synovial fibroblasts. Biochem Biophys Res Commun. 2004;324(1):3-7. doi:10.1016 j.bbrc.2004.09.011.

29. McLaughlin LM, Demple B. Nitric oxide-induced apoptosis in lymphoblastoid and fibroblast cells dependent on the phosphorylation and activation of p53. Cancer research. 2005;65(14):6097-6104. http://cancerres.aacrjournals.org /content/65/14/6097.full.pdf. Accessed October 2, 2013.

30. Pfeilschifter J, Rob P, Mülsch A, et al. Interleukin $1 \beta$ and tumour necrosis factor $\alpha$ induce a macrophage-type of nitric oxide synthase in rat renal mesangial cells. Eur J Biochem. 1992;203(1-2):251-255. doi:10.1111/j.1432-1033.1992.tb19854.x.

31. Zhang HY, Gharaee-Kermani M, Phan SH. Regulation of lung fibroblast $\alpha$-smooth muscle actin expression, contractile phenotype, and apoptosis by IL-1ß. J Immunol. 1997;158(3): 1392-1399.

32. Hemish J, Nakaya N, Mittal V, Enikolopov G. Nitric oxide activates diverse signaling pathways to regulate gene expression. J Biol Chem. 2003;278(43):42321-42329. doi:10.1074/jbc .M308192200.

33. Francis SH, Busch JL, Corbin JD, Sibley D. cGMP-dependent protein kinases and cGMP phosphodiesterases in nitric oxide and cGMP action. Pharmacol Rev. 2010;62(3):525-563. doi:10.1124 /pr.110.002907.
34. Bornancin F, Parker PJ. Phosphorylation of threonine 638 critically controls the dephosphorylation and inactivation of protein kinase Ca. Curr Biol. 1996;6(9):1114-1123. doi:10.1016/S09609822(02)70678-7.

35. Zaragoza C, Soria E, López E, et al. Activation of the mitogen activated protein kinase extracellular signal-regulated kinase 1 and 2 by the nitric oxide-cGMP-cGMP-dependent protein kinase axis regulates the expression of matrix metalloproteinase 13 in vascular endothelial cells [published correction appears in Mol Pharmacol. 2002;62(5):1259]. Mol Pharmacol. 2002;62(4): 927-935. http://molpharm. aspetjournals.org/content/62/4/927 .full.pdf. Accessed October 2, 2013.

36. Kubicek M, Pacher M, Abraham D, Podar K, Eulitz M, Baccarini M. Dephosphorylation of Ser-259 regulates Raf-1 membrane association. J Biol Chem. 2002;277(10):7913-7919. doi:10.1074 /jbc.M108733200.

37. Boulton TG, Nye SH, Robbins DJ, et al. ERKs: a family of protein-serine/threonine kinases that are activated and tyrosine phosphorylated in response to insulin and NGF. Cell. 1991;65(4):663-675. doi:10.1016/0092-8674(91)90098-J.

38. Mebratu Y, Tesfaigzi Y. How ERK1/2 activation controls cell proliferation and cell death: is subcellular localization the answer [review]? Cell Cycle. 2009;8(8):1168-1175. http: //www.landesbioscience.com/journals/cc/MebratuCC8-8.pdf. Accessed October 2, 2013.

39. Tejedo JR, Cahuana GM, Ramírez R, et al. Nitric oxide triggers the phosphatidylinositol 3-kinase/Akt survival pathway in insulin-producing RINm5F cells by arousing Src to activate insulin receptor substrate-1. Endocrinology. 2004;145(5):2319-2327. doi:10.1210/en.2003-1489.

40. Kennedy SG, Wagner AJ, Conzen SD, et al. The PI 3-kinase/Akt signaling pathway delivers an anti-apoptotic signal. Genes Dev. 1997;11(6):701-713. doi:10.1101/gad.11.6.701.

41. Vivanco I, Sawyers CL. The phosphatidylinositol 3-Kinase AKT pathway in human cancer. Nat Rev Cancer. 2002;2(7):489-501. doi:10.1038/nrc839.

๑) 2013 American Osteopathic Association 